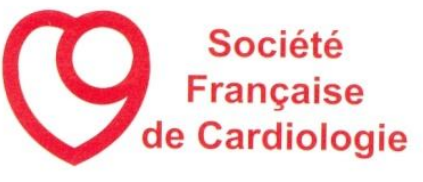

31 èmes Journées de I'hypertension artérielle

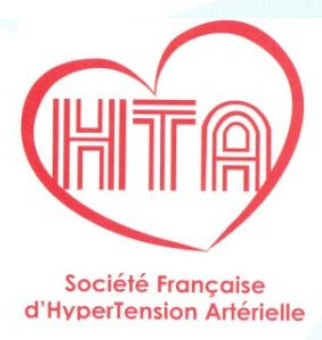

15 -16 décembre 2011

Palais des Congrès de Paris
ISSN 1261-694X

\section{archives des maladies}

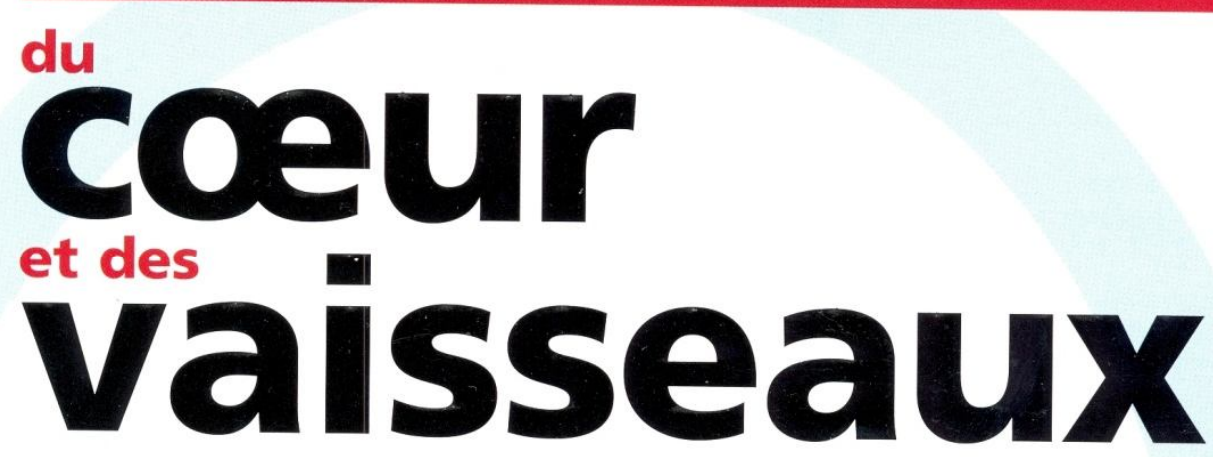

HORS-SERIE 3 - Décembre 2011 CO-58

\title{
RELATIONS ENTRE GAIN BARO-REFLEXE ET STRESS PULSATILE CHEZ LE PATIENT DIABETIQUE DE TYPE 1
}

André J. Scheen, M onique M archand, Jean-Christophe Philips (1)

(1) Service de Diabétologie, Nutrition et Maladies métaboliques, CHU Sart Tilman, Université de Liège,

\section{Objectifs :}

Liège, Belgique.

Le patient diabétique de type 1 (DT1) est exposé à un risque accru de neuropathie autonome cardiovasculaire (NAC) et de rigidité artérielle responsable d'une augmentation de la pression pulsée (PP). Le but du travail est d'étudier les relations entre la NAC (évaluée par le gain baro-réflexe ou GBR) et la rigidité artérielle (évaluée par le stress pulsatile ou SP) chez le patient DT1.

\section{Méthodes :}

Dans une étude transversale, GBR et SP ont été mesurés chez 167 patients DT1 soumis à un enregistrement continu de la pression artérielle (PA) et de la fréquence cardiaque (FC) lors d'un test postural standardisé (1min debout - 1min accroupi - 1min debout) et chez 160 sujets témoins appariés. GBR ( $\mathrm{msec} / \mathrm{mmHg}$ ) a été calculé par la pente de la relation entre les espaces $R-R$ et la PA systolique lors du redressement. SP $(\mathrm{mmHg} / \mathrm{min})$ a été calculé par le produit PPxFC durant les 3 min du test et en position accroupie. Dans une étude longitudinale, GBR et SP ont été évalués avant et après un suivi moyen de $79 \pm 33$ mois chez 74 patients (37 ans à l'inclusion, 19 années de DT1, HbA1c moyenne entre les deux tests : 8.6+1.5\%).

\section{Résultats :}

Dans l'étude transversale, GBR $(2,76 \pm 2,34$ versus $3,81 \pm 2,98 ; p<0,0005)$ et SP $(4862 \pm 1408$ versus 4229+1045; $p \varangle 0,0001)$ sont, respectivement, diminué et augmenté chez les DT1 par rapport aux témoins, avec une corrélation significative entre GBR et $S P(r=0,28 ; p=0,0003)$. Les DT1 avec un BRG abaissé $(n=85$; $1,17 \pm 0,61$ ) ont un SP accru ( 5190 vs $4521 ; p=0,0019$ ), et davantage encore en position accroupie (5408 vs 4396; $p \varangle 0,0001$ ), par comparaison aux DT1 avec un GBR élevé ( $n=82 ; 4,51+2,31)$. Dans l'étude longitudinale, une réduction significative du GBR est observée (de 3,34 $\pm 2,99$ à $2,47 \pm 2,08 ; p=0,0022$ ), mais

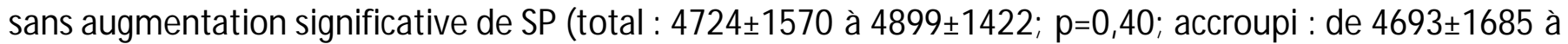
$4969+1515 ; p=0,29)$. En analyse univariée, les variations du GBR ne sont pas corrélées significativement avec le délai entre les deux mesures $(p=0,22)$, la durée du DT1 $(p=0,72)$ et le taux moyen d'HbA1c $(p=0,91)$, mais bien avec les variations de SP $(r=0,43 ; p=0,0003)$.

\section{Conclusion :}

Chez le patient DT1, GBR est diminué et SP est augmenté par rapport à une population témoin appariée pour l'âge. II existe une corrélation entre les marqueurs de la NAC et de la rigidité artérielle, mais GBR semble se détériorer plus rapidement que SP chez le patient DT1de la quarantaine imparfaitement équilibré. 